\title{
Intermediate Standstill Clones Trapped in the Reprogramming of Human Fibroblasts to Induced Pluripotent Stem Cells
}

\author{
Lifei Zhang, ${ }^{1}$ Yebo Wang, ${ }^{2}$ Ye Zhang, ${ }^{1}$ Limengmeng Wang, ${ }^{3}$ and He Huang ${ }^{3}$
}

\begin{abstract}
Factor-induced reprogramming of somatic cells into induced pluripotent stem cells (iPSCs) as a powerful tool for regenerative medicine has gained wide attention in recent years. However, there are certain concerns regarding the efficiency of this reprogramming. Partially reprogrammed iPSCs (piPSCs) are stable cell lines originating from cells that have exited the normal reprogramming route at an early time point. Analysis of the associated global gene expression changes between iPSCs and piPSCs may help understand the barriers to reprogramming. In our study, human fibroblasts were transduced with the four classic transcription factors, $O C T 4, S O X 2, K L F 4$, and $C-M Y C$. Only a few cells were completely reprogrammed to a fully pluripotent state. Instead, we obtained more number of intermediate standstill clones than human-induced pluripotent stem cells (hiPSCs) during reprogramming. We studied the genome-wide expression profiles of two different fibroblasts, five intermediate standstill clones, and two iPSCs derived from the two fibroblasts. Hierarchical clustering and principal component analysis demonstrated that intermediate standstill clones were on the way to becoming hiPSCs. A remarkable difference in the expression of genes related to cancer and cell adhesion pathway was observed between the intermediate standstill clones and iPSCs. These observations suggest that some cells may become trapped in partially reprogrammed states.
\end{abstract}

Keywords: reprogramming, iPS, intermediate standstill clones

\section{Introduction}

$\mathbf{F}$ ACTOR-INDUCED REPROGRAMMING of somatic cells into induced pluripotent stem cells (iPSCs) as a powerful tool for regenerative medicine has gained wide attention in recent years (Takahashi et al., 2007; Yu et al., 2007). These cells can potentially be used for cell therapy, and act as a model to study human disease and development, as well as drug screening and toxicology.

However, there are certain concerns regarding reprogramming, including its low efficiency, heterogeneity of most methods, and uncontrollability of the reprogramming process. Owing to these limitations, some studies are primarily focused on the immediate response of somatic cells to factor expression. For instance, fibroblasts were shown to undergo a process that was reminiscent of a mesenchymal-to-epithelial transition within a few days of OKSM expression (Li et al., 2010; Samavarchi-Tehrani et al., 2010). Some research groups, which are engaged in investigating the roadmap and identifying roadblocks during reprogramming, have reported that fibroblasts undergoing reprogramming pass through a number of defined intermediates (Brambrink et al., 2008; Stadtfeld et al., 2008).

Understanding the biological mechanisms underlying successful iPSC generation requires both accurate capture of cells undergoing the reprogramming process and identification of the associated global gene expression changes. In our study, human fibroblasts were transduced with the four classic transcription factors, OCT4, SOX2, KLF4, and C$M Y C$. Only a few cells were completely reprogrammed to a fully pluripotent state. Instead, we obtained more number of intermediate standstill clones than human-induced pluripotent stem cells (hiPSCs) during reprogramming. These clones are on the way to becoming hiPSCs, but fail to do so and became immortal at the very beginning step.

\footnotetext{
${ }^{1}$ Sir Run Run Shaw Hospital, Zhejiang University School of Medicine, Hangzhou, China.

${ }^{2}$ National Center for Science and Technology Evaluation, Haidian District, China.

${ }^{3}$ Zhejiang University School of Medicine First Affiliated Hospital, Hangzhou, China.
} 
FIG. 1. Morphology of established hiPSCs and intermediate standstill clones. hiPSCs, human-induced pluripotent stem cells.

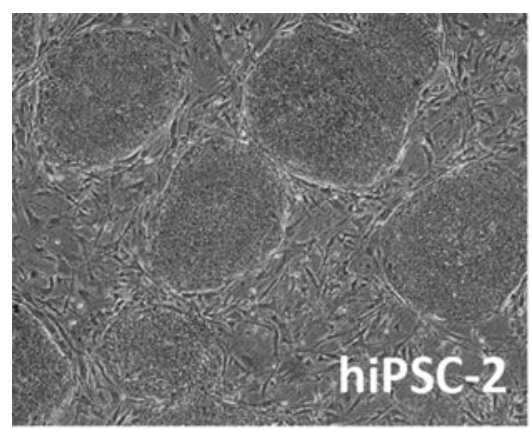

hiPSC (40x)

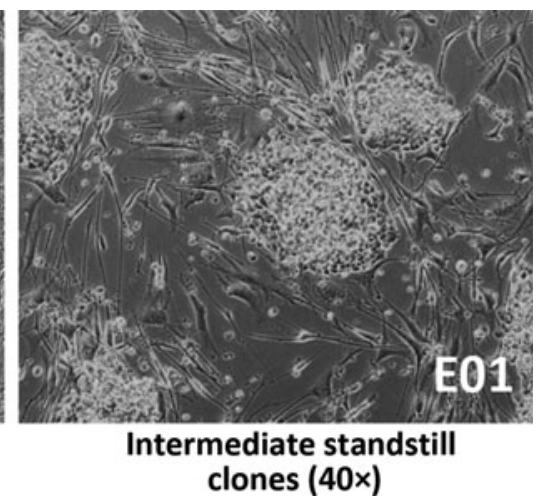

\section{Materials and Methods}

\section{Cell culture}

The same cell lines used in our previous study published in 2012 were used in this study (Zhang et al., 2013). Human foreskin fibroblasts were cultured in Eagle's minimum essential medium (Invitrogen) supplemented with $10 \%(\mathrm{v} / \mathrm{v})$ fetal bovine serum (FBS; Invitrogen). The fibroblasts were subcultured by treatment with $0.05 \%$ trypsin $/ 0.53 \mathrm{mM}$ EDTA (Invitrogen). $\mathrm{H} 1$ cells were obtained from the National Stem Cell Bank and were grown in human embryonic stem cells (hESCs) medium containing Dulbecco's modified Eagle's medium (DMEM)/F12 (Invitrogen) supplemented with $20 \%$ knockout serum replacement (Invitrogen), $0.1 \mathrm{mM}$ nonessential amino acids (Invitrogen), $1 \mathrm{mM}$ glutamine (Invitrogen), 0.1 mM 2-mercaptoethanol (Invitrogen), and $4 \mathrm{ng} / \mathrm{mL}$ basic fibroblast growth factor (bFGF; Invitrogen). The culture medium was changed daily.

The hiPSCs were cultured in a similar medium, except that the concentration of bFGF was increased to $10 \mathrm{ng} / \mathrm{mL}$. The hESCs and hiPSCs were passaged using $1 \mathrm{mg} / \mathrm{mL}$ of collagenase IV (Invitrogen). 293T cells were obtained from the American Type Culture Collection and were cultured in DMEM with high glucose (Invitrogen) supplemented with $10 \%$ FBS.

\section{Retrovirus production, infection, and iPSCs and intermediate standstill clones generation}

Moloney-based retroviral vectors (pMXs) containing the human genes encoding the four cell reprogramming factors, $O C T 4, S O X 2, K L F 4$, and $C-M Y C$, were obtained from Ad- dgene. Each plasmid was co-transfected into 293T cells with packaging plasmids pCMV-gp and pCMV-G (kindly provided by Professor Jing-Kuan Yee, City of Hope) through calcium phosphate co-precipitation (Peng et al., 2001). Viral supernatants were harvested 48 hours after transfection.

The human foreskin fibroblasts were infected twice in 2 days with the four retroviral vectors. The first day of infection of fibroblasts was designated as day 1 . On day 6 , the infected fibroblasts were harvested and plated onto inactivated feeder layers (Takahashi et al., 2007). On day 7, the medium was replaced with hESC medium. Valproic acid (at a working concentration of $2 \mathrm{mM}$; Sigma-Aldrich) (Huangfu et al., 2008) and vitamin C (at a working concentration of $25 \mu \mathrm{g} / \mathrm{mL}$; SigmaAldrich) (Esteban et al., 2010) were added to enhance cell reprogramming efficiency. Independent colonies were picked 3-4 weeks after infection and further expanded on inactivated feeder layers in hESC medium.

\section{$R N A$ isolation and reverse transcriptase polymerase chain reaction}

Total RNA was isolated using TRIzol reagent (Invitrogen). Total RNA $(0.5 \mu \mathrm{g})$ was used for cDNA synthesis, and $3.5 \mu \mathrm{L}$ of the $20 \mu \mathrm{L}$ reverse-transcribed product was amplified using the PrimeScript ${ }^{\mathrm{TM}}$ RT-PCR Kit (Takara). Some of the primer sequences used in our study were the same as those used by Takahashi et al. (2007), whereas the others have been listed as follows: Spalt-like transcription factor 2 (SALL2): 5'-GCTCCTGAAGCCAAAGAATG-3', 5'-GGT GCTCTGGTACTGGGTGT-3'; ZFP42 zinc finger protein (ZFP42): 5'-GGCAAAGACAAGACACCAGAA-3', 5'-CC
FIG. 2. Alkaline phosphatase staining of hiPSCs and intermediate standstill clones. Color images are available online.

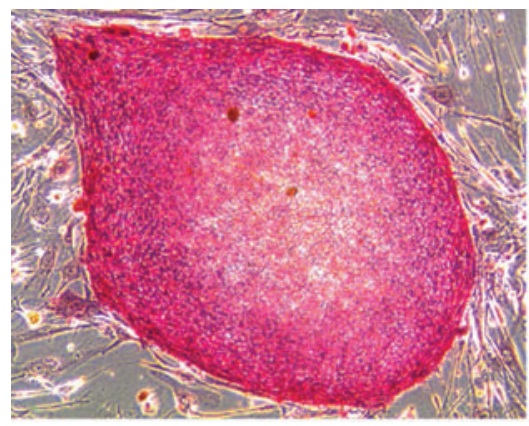

hiPSC (40x)

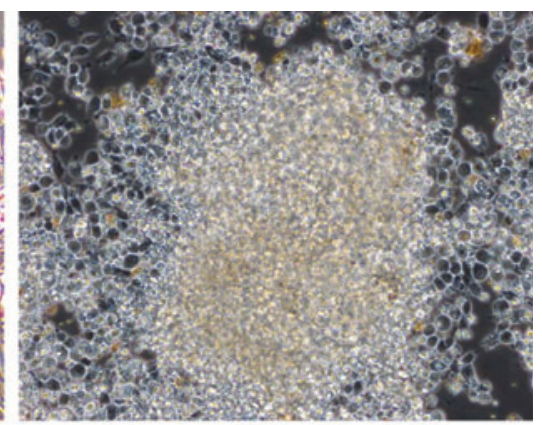

Intermediate standstill clones (100x) 
AGGATGGGTTGAGAAAACT-3'; transforming growth factor- $\beta$ (TGF- $\beta)$ : $5^{\prime}$-GTACCTGAACCCGTGTTGCT-3', 5'-GTCCTTGCGGAAGTCAATGT-3'.

\section{Alkaline phosphatase staining}

Alkaline phosphatase (AP) staining was performed using the Stemgent Alkaline Phosphatase Staining Kit (Stemgent). Cells were washed with phosphate-buffered saline (PBS) and fixed with fixing solution for 2 minutes at room tem-
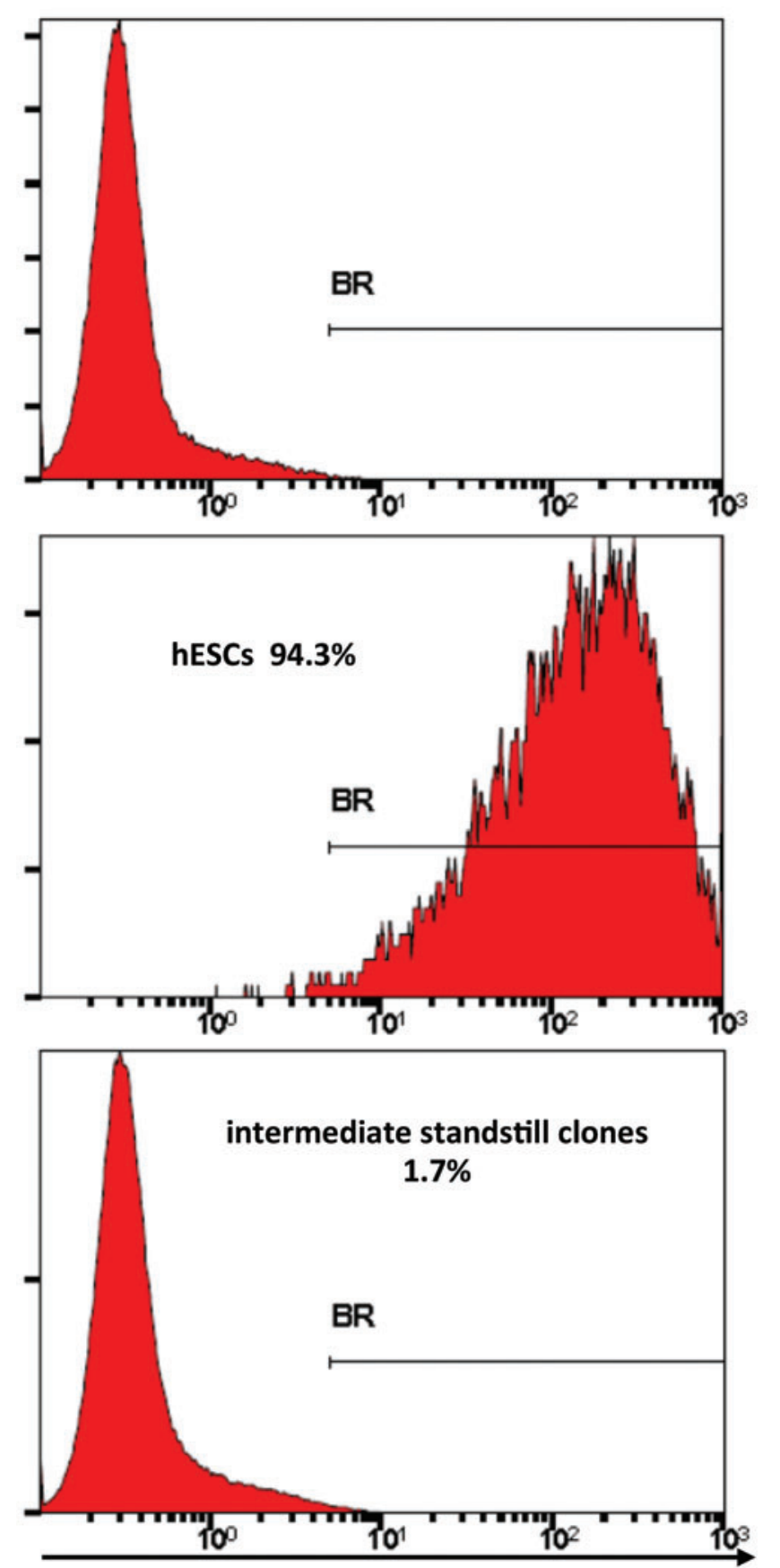

TRA-1-60

FIG. 3. FACS analysis of the expression of TRA-1-60 in hESCs and intermediate standstill clones. hESCs, human embryonic stem cells. Color images are available online. perature. Then fixing solution was removed. The cells were washed with PBS and treated with freshly prepared AP staining solution for 30 minutes at room temperature. Then AP staining solution was removed. The stained cells were washed twice with PBS.

\section{Genome-wide expression profiles}

Genome-wide expression profile analyses were carried out by GeneTech (Shanghai, China) Co. Ltd. Approximately $1 \times 10^{7}$ cells from each sample were collected for each GeneChip. Arrays were scanned with GeneChip PrimeView Human Gene Expression Array and GeneChip 3' IVT Express Kit, 1 reaction 901839-PART. Data were analyzed using Affymetrix Expression Array Data Analysis System (not included in the IVT kit).

\section{Statistical analysis}

All data are presented as mean \pm standard error of the mean. Differences between multiple groups were assessed by analysis of variance (ANOVA) using. The level of statistical significance was set to $p<0.05$.

\section{Results}

In our study, four classic transcription factors (OCT4, $S O X 2, K L F 4$, and $C-M Y C$ ) were transduced into human fibroblasts derived from two donors (F1 and F2). After 3-4 weeks, only a few cells were completely reprogrammed to a fully pluripotent state. Instead, we obtained more number of intermediate standstill clones than hiPSCs during reprogramming. These clones were not tightly packed and did not have sharp edges such as hiPSCs (Fig. 1). They proliferated much faster than hiPSCs (Theunissen et al., 2011).

Intermediate standstill clones Pre-A and Pre-B were derived from $\mathrm{F} 1$ fibroblasts. Intermediate standstill clones E01, F04, O05, M01, U02, O06, and E06 were derived from F2 fibroblasts. These clones were picked and cultured. After 710 passages, the intermediate standstill clones showed no $\mathrm{AP}$ activity, no expression of the hESC specific marker

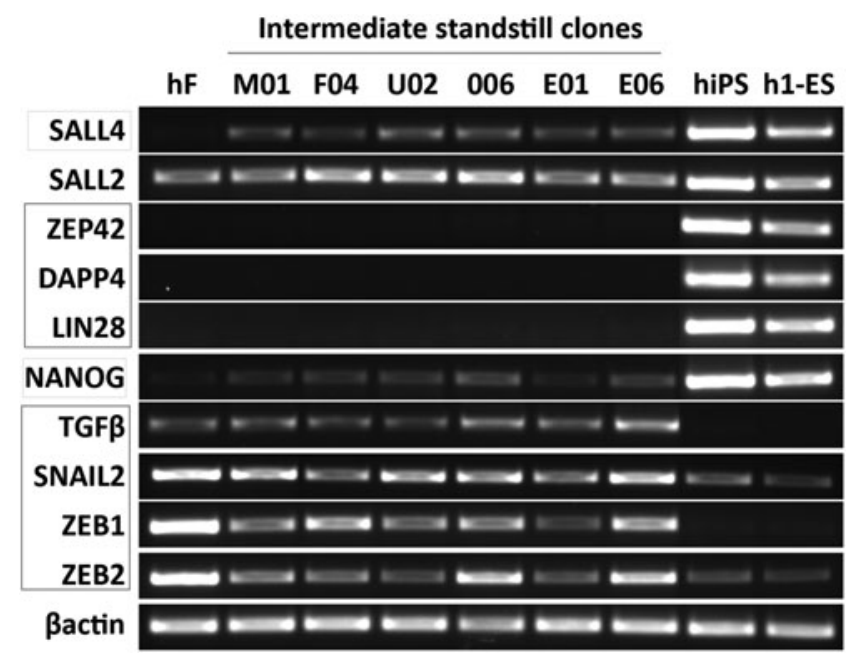

FIG. 4. Reverse transcriptase polymerase chain reaction analysis of the expression of hESCs marker genes and linagespecific genes in hiPSCs and intermediate standstill clones. 


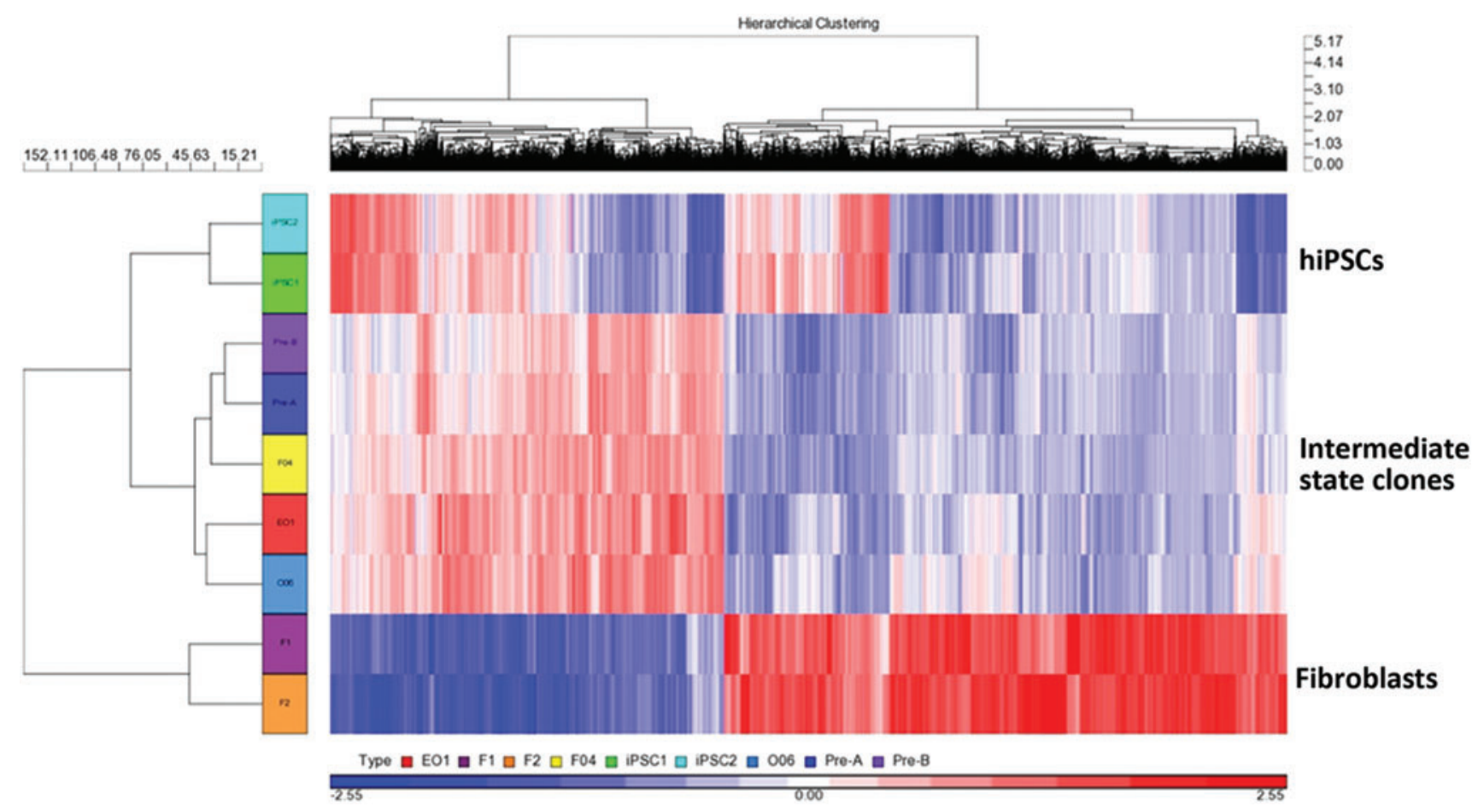

FIG. 5. Hierarchical clustering analysis of the genome-wide expression profiles in hiPSCs, intermediate standstill clones, and fibroblasts. Pre-A, Pre-B, and hiPSC1 derived from F1. E01, F04, O05, and hiPSC2 derived from F2. Color images are available online.

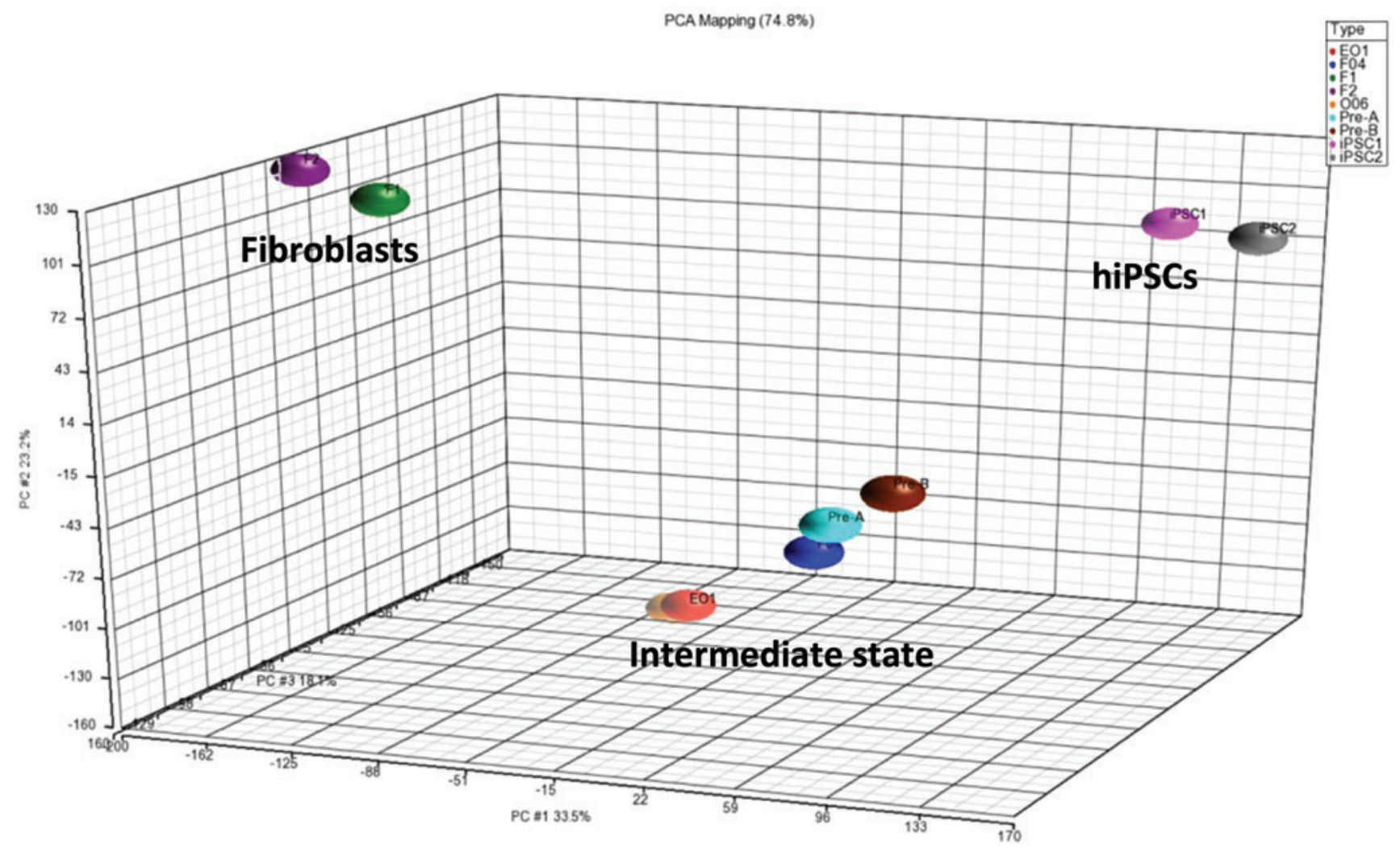

FIG. 6. PCA of the genome-wide expression profiles in hiPSCs, intermediate standstill clones and fibroblasts. PCA, principal component analysis. Color images are available online. 


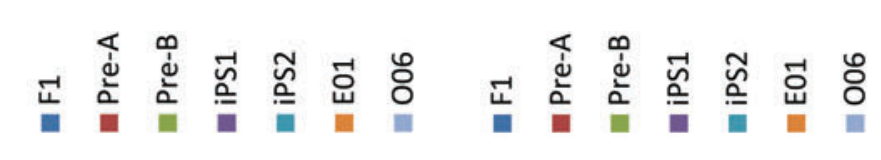
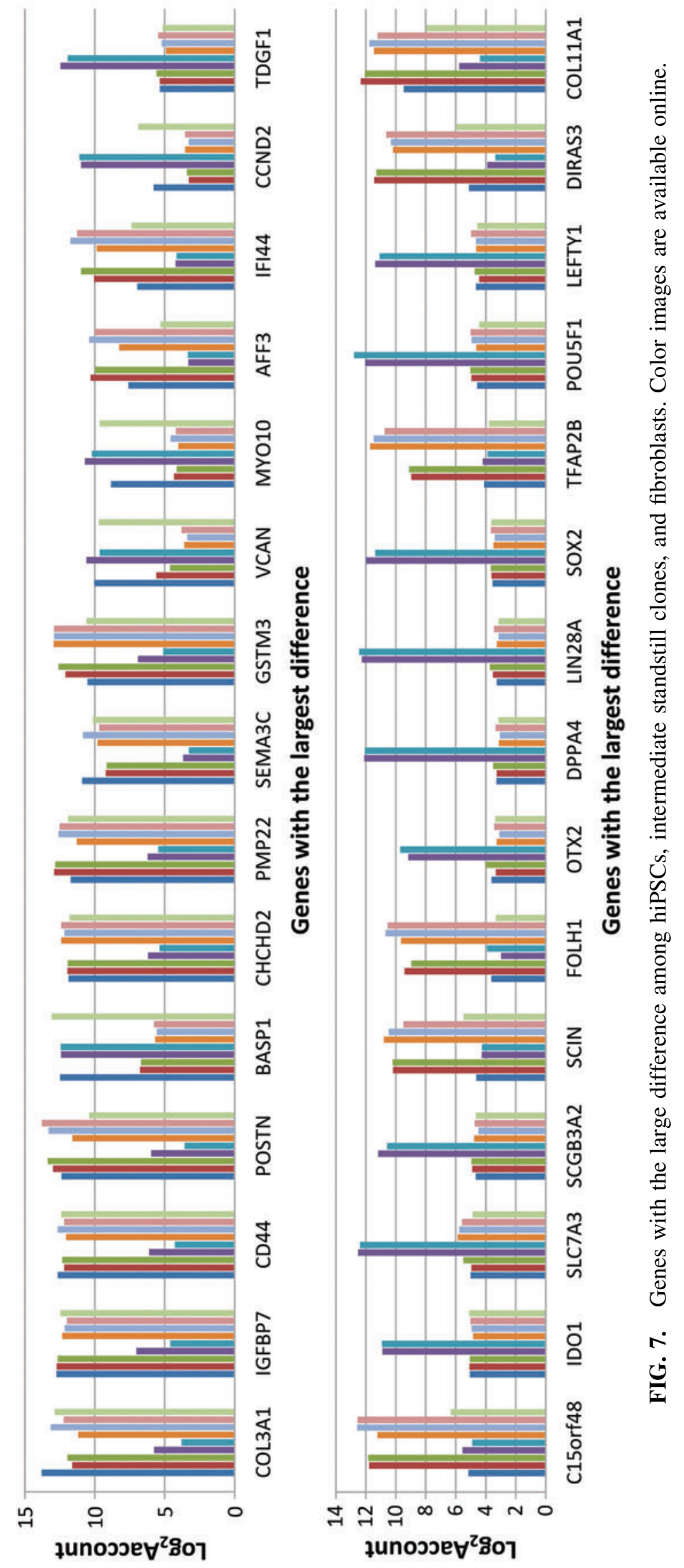
TRA-1-60 (Figs. 2 and 3), no expression of certain pluripotency-related genes (ZEP42, developmental pluripotency associated 4 [DAPP4], and lin-28 homolog A [LIN28]), and low expression of SALL4 and NANOG. More importantly, they continued to express several lineage-specific genes $(T G F-\beta$, snail family transcriptional repressor 2 [SNAIL2], zinc finger E-box binding homeobox 1 [ZEB1], and $Z E B 2$ ), which are supposed to be silenced (or downregulated) at an early stage of reprogramming (Fig. 4).

To identify the possible obstacles during reprogramming, we studied and compared the genome-wide expression profiles of $\mathrm{F} 1$ and $\mathrm{F} 2$ fibroblasts, five intermediate standstill clones (E01, F04, O05, Pre-A, and Pre-B), and two hiPSCs derived from the two fibroblasts (hiPSC1, Pre-A and Pre-B derived from F1. hiPSC2, E01, F04 and O05derived from F2 fibroblasts). Hierarchical clustering and principal component analysis (PCA) demonstrated that the intermediate standstill clones displayed a highly similar (but distinct) molecular makeup, but were not identical to both original fibroblasts and hiPSCs (Figs. 5 and 6). They were on the way to becoming hiPSCs, but failed to do so and became immortal at the very beginning step, which is in accordance with the results of the study by Polo et al. (2012).

Furthermore, we analyzed the difference in gene expression between hiPSCs, intermediate standstill clones, and fibroblasts. A marked difference in the expression of several genes related to cancer and cell adhesion pathway was observed between the intermediate standstill clones and hiPSCs, indicating that they may play an important role in the reprogramming process. The difference in expression was highest for the genes periostin (POSTN), DIRAS family GTPase 3 (DIRAS3), interferon-induced protein 44 (IFI44), and scinderin (SCIN), which are related to the p53, Erk, and AKT signaling pathways (Fig. 7).

\section{Discussion}

Some researches in mouse have reported that lineagecommitted cells show a complex response to the ectopic expression of the defined transcription factors and some stable intermediate stages emerge (Mikkelsen et al., 2008). These intermediate stages cell lines (known as partially iPSCs) show reactivation of some stem-cell-related genes, incomplete repression of lineage-specifying transcription factors, incomplete epigenetic remodeling, and originate from cells that have exited the normal reprogramming route at an early time point, hence showing little overlap with progressing intermediates (Mikkelsen et al., 2008).

One study (Polo et al., 2012) comprehensively analyzed the transcriptional and epigenetic changes in the phenotypically defined intermediates of iPSC induction, and concluded that failure to activate certain ESC-specific genes acts as a roadblock to reprogramming. In our study, we obtained some intermediate standstill clones during human fibroblasts reprogramming. The intermediate standstill clones showed low expression of SALLA and NANOG and no expression of certain pluripotency-related genes. They continued to express several lineage-specific genes. The gene expression profile of the intermediate standstill clones displayed a highly similar (but distinct) molecular makeup, but was not identical to both original human fibroblasts and hiPSCs. A marked difference in the expression of genes related to cancer and cell adhesion pathway was observed between the intermediate standstill clones and hiPSCs.

Cells may fail to reprogram successfully for several apparent reasons. They may inappropriately activate or fail to repress endogenous or ectopic transcription factors. In further experiments, we need to detect the expression of endogenous and exogenous $O C T 4, S O X 2, K L F 4$, and $C-M Y C$. They may fail to be infected by all four factors. The reprogramming method used in our study did not provide high efficiency in hiPSC regeneration. The five intermediate standstill clones appear to spread out more than hiPSCs or fibroblasts, which are more clustered in PCA of genomewide expression profile. These data suggest that the different profile may be due to different levels of retroviral transduction efficiency.

Furthermore, complete reprogramming can be facilitated by direct intervention against these failure modes. For example, a previous study (Theunissen et al., 2011) showed that OCT4 overexpression inhibits pluripotency gene expression and $N A N O G$ has the capacity to overcome the reprogramming block in partially iPSCs.

In further study, we expect to claim whether or not these differentially expressed genes or pathways are roadblocks of reprogramming. And to provide a powerful tool for defining these states and increasing the efficiency of inducing mature cells back to a pluripotent state by treating them with specific pathway-related molecules during the reprogramming process.

\section{Author Disclosure Statement}

The authors declare that no conflicting financial interests exist.

\section{Funding Information}

This study was supported by National Natural Science Foundation of China (NSFC) grants (81000198 and 81600118), and Zhejiang Provincial Natural Science Foundation of China (LQ17H080002).

\section{References}

Brambrink, T., Foreman, R., Welstead, G.G., Lengner, C.J., Wernig, M., Suh, H., and Jaenisch, R. (2008). Sequential expression of pluripotency markers during direct reprogramming of mouse somatic cells. Cell Stem Cell 2, 151-159.

Esteban, M.A., Wang, T., Qin, B., Yang, J., Qin, D., Cai, J., Li, W., Weng, Z., Chen, J., Ni, S., Chen, K., Li, Y., Liu, X., Xu, J., Zhang, S., Li, F., He, W., Labuda, K., Song, Y., Peterbauer, A., Wolbank, S., Redl, H., Zhong, M., Cai, D., Zeng, L., and Pei, D. (2010). Vitamin C enhances the generation of mouse and human induced pluripotent stem cells. Cell Stem Cell 6, 71-79.

Huangfu, D., Maehr, R., Guo, W., Eijkelenboom, A., Snitow, M., Chen, A.E., and Melton, D.A. (2008). Induction of pluripotent stem cells by defined factors is greatly improved by small-molecule compounds. Nat. Biotechnol. 26, 795-797.

Li, R., Liang, J., Ni, S., Zhou, T., Qing, X., Li, H., He, W., Chen, J., Li, F., Zhuang, Q., Qin, B., Xu, J., Li, W., Yang, J., Gan, Y., Qin, D., Feng, S., Song, H., Yang, D., Zhang, B., Zeng, L., Lai, L., Esteban, M.A., and Pei, D. (2010). A mesenchymal-to-epithelial transition initiates and is required for the nuclear reprogramming of mouse fibroblasts. Cell Stem Cell 7, 51-63. 
Mikkelsen, T.S., Hanna, J., Zhang, X., Ku, M., Wernig, M., Schorderet, P., Bernstein, B.E., Jaenisch, R., Lander, E.S., and Meissner, A. (2008). Dissecting direct reprogramming through integrative genomic analysis. Nature 454, 49-55.

Peng, H., Chen, S.T., Wergedal, J.E., Polo, J.M., Yee, J.K., Lau, K.H., and Baylink, D.J. (2001). Development of an MFGbased retroviral vector system for secretion of high levels of functionally active human BMP4. Mol. Ther. 4, 95-104.

Polo, J.M., Anderssen, E., Walsh, R.M., Schwarz, B.A., Nefzger, C.M., Lim, S.M., Borkent, M., Apostolou, E., Alaei, S., Cloutier, J., Bar-Nur, O., Cheloufi, S., Stadtfeld, M., Figueroa, M.E., Robinton, D., Natesan, S., Melnick, A., Zhu, J., Ramaswamy, S., and Hochedlinger, K. (2012). A molecular roadmap of reprogramming somatic cells into iPS cells. Cell 151, 1617-1632.

Samavarchi-Tehrani, P., Golipour, A., David, L., Sung, H.K., Beyer, T.A., Datti, A., Woltjen, K., Nagy, A., and Wrana, J.L. (2010). Functional genomics reveals a BMP-driven mesenchymal-to-epithelial transition in the initiation of somatic cell reprogramming. Cell Stem Cell 7, 64-77.

Stadtfeld, M., Maherali, N., Breault, D.T., and Hochedlinger, K. (2008). Defining molecular cornerstones during fibroblast to iPS cell reprogramming in mouse. Cell Stem Cell 2, 230-240.

Takahashi, K., Tanabe, K., Ohnuki, M., Narita, M., Ichisaka, T., Tomoda, K., and Yamanaka, S. (2007). Induction of plurip- otent stem cells from adult human fibroblasts by defined factors. Cell 131, 861-872.

Theunissen, T.W., van Oosten, A.L., Castelo-Branco, G., Hall, J., Smith, A., and Silva, J.C. (2011). Nanog overcomes reprogramming barriers and induces pluripotency in minimal conditions. Curr. Biol. 21, 65-71.

Yu, J., Vodyanik, M.A., Smuga-Otto, K., Antosiewicz-Bourget, J., Frane, J.L., Tian, S., Nie, J., Jonsdottir, G.A., Ruotti, V., Stewart, R., Slukvin, I.I., and Thomson, J.A. (2007). Induced pluripotent stem cell lines derived from human somatic cells. Science 318, 1917-1920.

Zhang, L., Zheng, W., Wang, Y., Wang, Y., and Huang, H. (2013). Human bone marrow mesenchymal stem cells support the derivation and propagation of human induced pluripotent stem cells in culture. Cell. Reprogram. 15, 216-223.

Address correspondence to:

Lifei Zhang

Sir Run Run Shaw Hospital Zhejiang University School of Medicine

Hangzhou 310016

China

E-mail: zhanglf@zju.edu.cn 